\title{
Gain Enhancement of Millimeter-Wave on-Chip Antenna through an Additively Manufactured Functional Package
}

\author{
Haoran Zhang, Student Member, IEEE, and Atif Shamim, Senior Member, IEEE
}

\begin{abstract}
Antenna-on-chip (AoC) presents an excellent solution for applications requiring higher integration levels and lower cost, however, its radiation performance is poor due to the inherent lossy Si substrate in conventional complementary metaloxide-semiconductor (CMOS) processes. It is well known that every chip requires a package to protect it from the environment, however this package does not provide any functionality, despite adding to the cost and required space. In this paper, we propose a functional package which has been designed to enhance the gain of the AoC. A $71 \mathrm{GHz}$ coplanar waveguide (CPW) fed on-chip monopole antenna, driven by a voltage-controlled oscillator (VCO) and frequency doubler, was realized in standard $0.18 \mu \mathrm{m}$ CMOS technology. As a first step to enhance its gain, the AoC was designed on top of an Artificial Magnetic Conductor (AMC) surface. Further gain enhancement was achieved by transforming the chip package into a combination of a superstrate layer and a Fresnel lens, qualifying this as a System-on-Package (SoP). The package has been realized through additive manufacturing to maintain the low-cost aspect. Overall, the measured gain of the packaged $\mathrm{AoC}$ is $6.8 \mathrm{dBi}$, which has been enhanced by $\sim 20 \mathrm{dBs}$ as compared to the unpackaged antenna without an AMC layer.
\end{abstract}

Index Terms-Antenna-on-chip (AoC), Artificial Magnetic Conductor (AMC), superstrate, Fresnel lens, System-on-Package (SoP), gain enhancement

\section{INTRODUCTION}

$\mathrm{W}$ ITH the availability of millimeter-wave (mm-wave) bands, there has been a surge in wireless communication applications [1,2]. The multi-GHz of available bandwidth at the mm-wave bands makes it an ideal candidate for high data rate wireless applications [3]. Moreover, the wavelength at these frequency bands is in the range of $1-10 \mathrm{~mm}$, which is small enough to realize the AoC [4-8]. Integrating the $\mathrm{AoC}$ and integrated circuits (ICs) in standard CMOS processes can reduce the number of off-chip components in the system as well as eliminate the uncertain influence from the bond wires between the antenna and the ICs. However, the conventional AoC usually suffers from low gain and low radiation efficiency due to the lossy Si substrate in standard CMOS technology. Fig. 1 shows the stack up of a standard CMOS process. One of the main challenges is the low resistivity $(10-15 \Omega-\mathrm{cm})$ of the

The authors are with the Department of Electrical Engineering, King Abdullah University of Science and Technology, Thuwal 23955-6900, Saudi Arabia (e-mail: Haoran.zhang@kaust.edu.sa).
Si substrate used in standard CMOS technology. The low resistivity can avoid latch-up in the IC design [9], but in the AoC design, it causes most of the radio-frequency (RF) power to be absorbed by the Si rather than being radiated by the AoC. In addition, due to the high relative permittivity $\left(\varepsilon_{r}=11.9\right)$ and relatively large electrical thickness of the $\mathrm{Si}$, high order surface wave modes are excited, which further degrades the antenna's radiation performance [2]. Another challenge is the mutual coupling between the AoC and the nearby metals in the circuit (such as the interconnection wires, inductors, capacitors, and dummy metals), which can affect current distribution in the AoC. As a result, the radiation performance and impedance matching are further deteriorated [10].

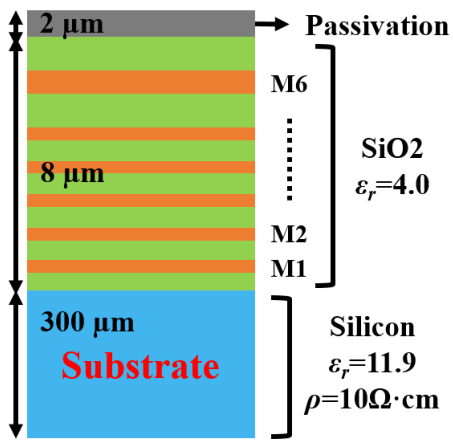

Fig. 1. Standard CMOS stack up

To overcome these challenges, several solutions have been proposed [11-20]. One solution is to alter the structure of the lossy Si substrate through micromachining to reduce the $\mathrm{Si}$ thickness $[11,16,20]$. In [20], an air cavity was formed at the backside of the Si substrate underneath the AoC, so the losses in the $\mathrm{Si}$ substrate were reduced because of the thinner substrate. Another option is to implant protons in the $\mathrm{Si}$ substrate to increase its resistivity, which can decrease the RF power absorption in the Si $[12,19]$. Aside from changing the properties of the lossy Si substrate, a micro-electromechanical system (MEMS) structure has been used for gain enhancement [18]. By applying a DC control voltage, the on-chip bowtie antenna can be lifted to move away from the lossy $\mathrm{Si}$, thus achieving gain enhancement. Although micromachining, proton implantation, and MEMS structures can successfully improve the $\mathrm{AoC}$ radiation performance, they are not compatible with standard CMOS processes and require significantly more complicated post-fabrication processes. 
Alternatively, the AMC surface can be applied to isolate the AoC from the lossy Si substrate. For example, in $[3,14,15]$, the AMC surfaces were realized using the metal layers in the CMOS stack up to enhance the AoC gain. In-phase reflection occurs when electromagnetic (EM) waves from the AoC are incident on the AMC surface, so the boresight radiation can be boosted by the constructive superposition of the AoC's direct radiation and in-phase reflection. In addition to the on-chip gain enhancement approaches, some studies have used off-chip structures to enhance the AoC radiation performance $[13,17]$.

An interesting approach to enhance the AoC gain is to functionalize the chip package since every chip needs a package for protection from the environment. However, typically, these packages are mere protectors and do not have any functionality, despite adding to the cost and required space. In this work, we introduce an SoP design concept, where the chip package has been optimized to work as a superstrate layer as well as a Fresnel lens, thus enhancing the antenna's gain in addition to providing the required protection to the chip. Overall, the AoC with the functional package demonstrated a gain enhancement of $\sim 20 \mathrm{dBs}$ compared to the unpackaged AoC. The initial simulation results of the on-chip antenna with a superstrate are shown in [21]. This paper covers the detailed design steps for the on-chip antenna with an AMC, the enhancement of gain through a functional package comprising of a superstrate and Fresnel lens, the additive manufacturing of the package, the measured results of the standalone chip, and the complete SoP. The results of this work are promising and indicate that a compact and low-cost AoC, which was previously only useful for very short-range applications, can also be used for medium or even longer range applications by smartly packaging the ICs.

The paper is organized as follows: Section II presents the design of the on-chip AMC structure. Section III shows the detailed design for the functional package and resultant gain enhancement in simulations. Section IV briefly describes the antenna's driving circuits. Section V presents the fabrication of the functional package and the overall measured results.

\section{ON-CHIP AMC SURFACE AS A REFLECTOR}

The standard $0.18 \mu \mathrm{m}$ CMOS process consists of a $6-10 \mu \mathrm{m}$ thick inter-metal dielectric embedding 6-9 metal layers, as shown in Fig. 1. The AoC is usually implemented on the top metal layer because this layer provides sufficient metal thickness for the realization of the AoC. Moreover, it helps direct the radiation into the free space. A standalone quarter-wavelength monopole antenna has been simulated with the CMOS stack-up. However, only $-13.6 \mathrm{dBi}$ antenna gain is obtained because of the lossy Si substrate. Ideally, in order to isolate the $\mathrm{AoC}$ from the lossy Si substrate, a Perfect Electrical Conductor (PEC) surface could be placed on the Metal 1 (M1) layer. However, in this case, the PEC surface becomes electrically too close to the $\mathrm{AoC}$ where the distance is around 7 $-8 \mu \mathrm{m}$, as a result, the induced image current on the PEC can negatively affect the antenna's radiation performance. According to the boundary conditions, a Perfect Magnetic
Conductor (PMC) surface can resolve this issue because the PMC surface can have an in-phase reflection rather than the out-of-phase reflection from a PEC surface. Since no PMC surface exists in nature, an AMC surface, which is a combination of a periodic structure and a ground plane (Fig. 2 (a)), was proposed to mimic a PMC surface within a specific frequency band.

In this work, the AMC structure consists of a square patch-based periodic frequency selective surface on the M1 layer and a ground plane at the backside of the Si substrate as shown in Fig. 2 (a). The square patch was selected as the AMC unit cell because it has larger bandwidth and lower substrate loss compared to other basic AMC unit cell structures [22].

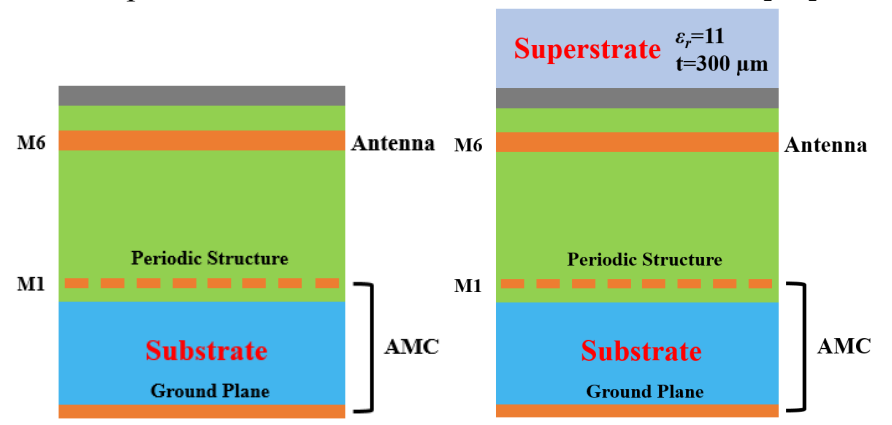

(a)

(b)

Fig. 2. Stack up of (a) the AoC integrated with the AMC structure, and (b) the AoC integrated with the AMC and superstrate

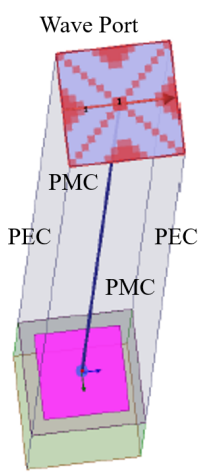

(a)

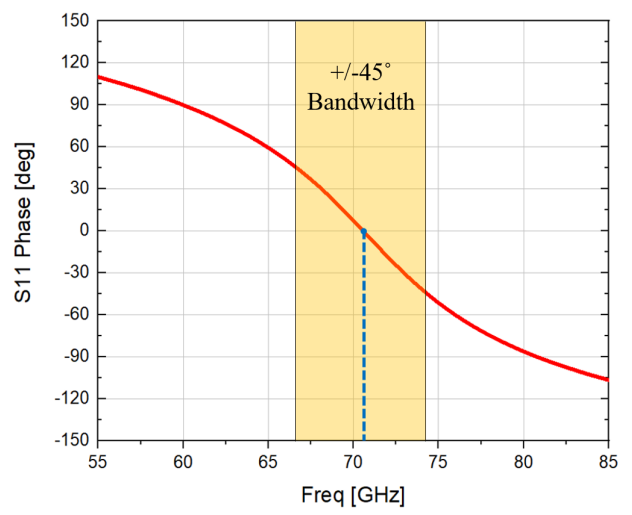

(b)
Fig. 3. (a) High Frequency Simulation Sotfware (HFSS) model for an AMC cell and (b) reflection phase of the AMC structure

Fig. 3 (a) shows the simulation model of the square patch AMC unit cell in HFSS. To mimic the infinite AMC surface, PEC and PMC boundary wall conditions were used in the simulations, as shown in Fig. 3 (a). The wave port was assigned on the top of the square patch at a distance of quarter wavelength. Later, the wave port was de-embedded to the surface of the AMC surface to eliminate the additional transmission phase shift [21]. The dimension of the square patch was optimized to achieve the in-phase reflection at 71 $\mathrm{GHz}$, as shown in Fig. 3 (b). A bandwidth of $7.4 \mathrm{GHz}$ was achieved, in which the AMC operating bandwidth is defined as the frequency range within $+/-45^{\circ}$ of the phase of the reflection coefficient.

In theory, the AMC surface needs to be infinite to behave as 
a PMC surface, however, this has not been possible to realize on a very small chip. In order to determine a reasonable size for the AMC structure, a CPW fed quarter-wavelength monopole antenna was simulated on top of varying AMC surface sizes. Fig. 4 illustrates that, as expected, the gain of the on-chip monopole was enhanced with the increasing number of AMC unit cells. However, the AMC surface size is restricted by the chip size, which is $1.5 \mathrm{~mm} \times 1.5 \mathrm{~mm}$. As a tradeoff between size and maximum antenna gain, an AMC size of $6 \times 8$ unit cells $\left(0.48 \mathrm{~mm}^{2}\right)$ was selected.

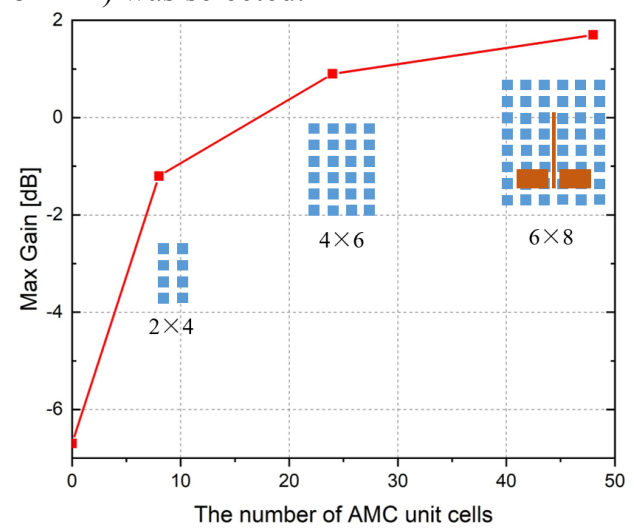

Fig. 4. Maximum gain of a monopole antenna versus the AMC surface size

\section{FunCTIONAL CHIP PACKAGE FOR GAIN ENHANCEMENT}

\section{A. Superstrate Layer}

A superstrate with a high permittivity can increase the antenna's coupling into free space, thus improving the boresight radiation and reducing substrate losses as less EM waves are absorbed in the lossy $\mathrm{Si}$ substrate. Fig. 2 (b) demonstrates the superstrate integration with the AMC-backed AoC. In this design, the superstrate material is PREFERM FLX 1100 , which has a high permittivity of $\varepsilon_{r}=11$ and a low loss tangent of $\tan \delta=0.006$ at $71 \mathrm{GHz}$.

In order to investigate the effect of the superstrate thickness, a CPW-fed AoC (quarter-wavelength monopole antenna) was optimized for the best radiation efficiency for varying thicknesses of the superstrate. As Fig. 5 shows, maximum radiation efficiency was achieved with a superstrate thickness equal to $300 \mu \mathrm{m}$, while the second maximum was obtained with a superstrate thickness of around $900 \mu \mathrm{m}$. This result indicates that the radiation efficiency is maximized when the thickness of the superstrate layer is equal to odd multiples of $\lambda_{d} / 4$, where $\lambda_{d}$ is the wavelength in the medium. In terms of the transmission line equivalent model, the superstrate layer behaves as a quarter wavelength impedance transformer, thereby improving the antenna's coupling to free space [23]. Consequently, this design uses a superstrate thickness of $300 \mu \mathrm{m}$.

Since the superstrate is quite thick $\left(\lambda_{d} / 4\right)$, it is capable of providing good protection for the chip. Therefore, the superstrate can act as the chip package as well provide gain enhancement. In addition, no metal patterning or alignment is required during the superstrate preparation, so it is easy to realize the superstrate.

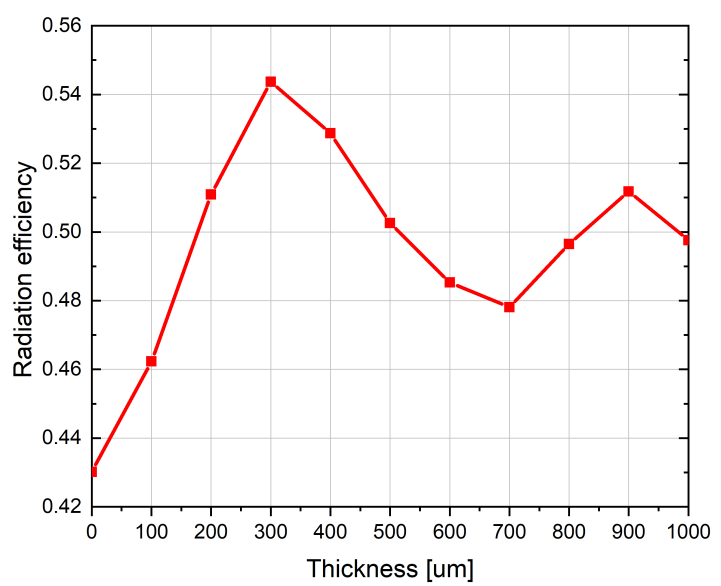

Fig. 5. Radiation efficiency versus superstrate thickness

\section{B. Fresnel Lens Package}

Further gain enhancement can be achieved by incorporating a lens. A Fresnel lens can be used for gain enhancement through beam shaping, which is realized by diffraction instead of refraction in the conventional hyperboloid lens [24]. A Fresnel lens is a phase-correcting lens that consists of a set of planar grooved circular plates which should be made of a low-loss material [25]. As Fig. 6 illustrates, the EM waves from the AoC located at the focal point propagate an additional distance $\Delta$ from the outer surface of the sphere to the edge of each zone plate.

$$
\Delta=n \times \frac{\lambda_{0}}{P}
$$

Where $n$ is the number of planar plates and $P$ is the number of different phases for realizing phase correction. For example, $P$ is equal to 2 and 4 in half-wavelength and quarter-wavelength phase-correcting lenses, respectively. The beam-focusing performance improves with a larger $P$.

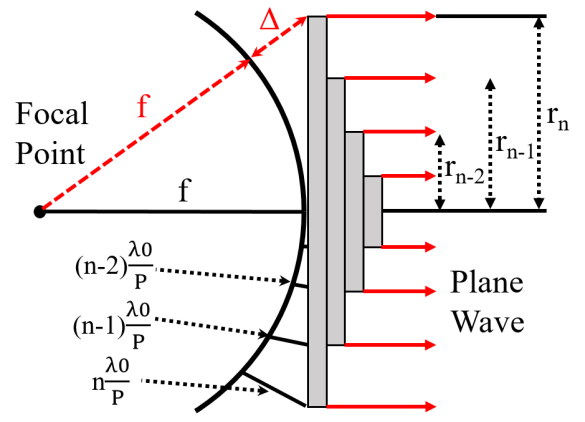

Fig. 6. Geometry of the wave propagation in a Fresnel lens package

For a quarter-wavelength phase-correcting lens, if the AoC is placed at the focal point, the EM waves radiated from the AoC will pass through different parts of the zone plates with less than a $90^{\circ}$ phase shift. Then, the plane wave is formed after passing through the lens, as shown in Fig. 6. Thus, the beam-focusing function is achieved by the phase-correcting zone plates. The radius of each zone plate, $r_{n}$, is calculated by the right triangle formula [26]. 


$$
r_{n}=\sqrt{2 f \frac{n \lambda_{0}}{P}+\left(\frac{n \lambda_{0}}{P}\right)^{2}}
$$

In this design, $P=4$ was chosen, which is a good compromise between the focusing ability of the Fresnel lens and the complexity of the lens fabrication. The focal length is equal to $2.7 \mathrm{~mm}$, resulting in a relatively low $F / D$ ratio of 0.21 but ensuring a compact SoP design. The quarter-wavelength lens was grooved into four-layer zone plates with depths of $d$, $2 d$, and $3 d$. The depth, $d$, is provided in [26].

$$
d=\frac{\lambda_{0}}{4\left(\sqrt{\varepsilon_{r}}-1\right)}
$$

Where $\varepsilon_{r}$ is the permittivity of the lens material. It is noted that the lens thickness is inversely proportional to the permittivity. Therefore, a high permittivity material (PREFERM 3D ABS filament, $\varepsilon_{r}=10, \tan \delta=0.02$ at $71 \mathrm{GHz}$ ) was chosen to ensure a compact design.

Fig. 7 (a) shows a cross-sectional view of the SoP design, which contains the AoC, on-chip AMC surface, functional package in the form of a superstrate, and Fresnel lens. A gap length of $f=2.7 \mathrm{~mm}$ was achieved through pillars constructed of the same packaging material. This gap helps maintain the required focal distance between the antenna and the lens. The combination of the superstrate and Fresnel lens not only provides protection from the environment, but it also provides gain enhancement.

The final simulation model also included the connection to the driving circuit output, as shown in Fig. 7 (b). In order to improve the accuracy of the simulation model, the circuit wires, inductors, capacitors, and dummy metals were all included in this simulation. Since this is the top view of the multilayered CMOS chip, only the top metal layer (M6) is clearly visible. The top layer of the circuit shows the VCO inductor $L_{l}$, the frequency doubler inductor $L_{2}$, the matching network inductors $L_{3}$ and $L_{4}$, ground-signal-ground (GSG) pads of the circuit RF output, and the three pads for the DC power supply. The detailed information of the circuit is presented in Fig. 10.

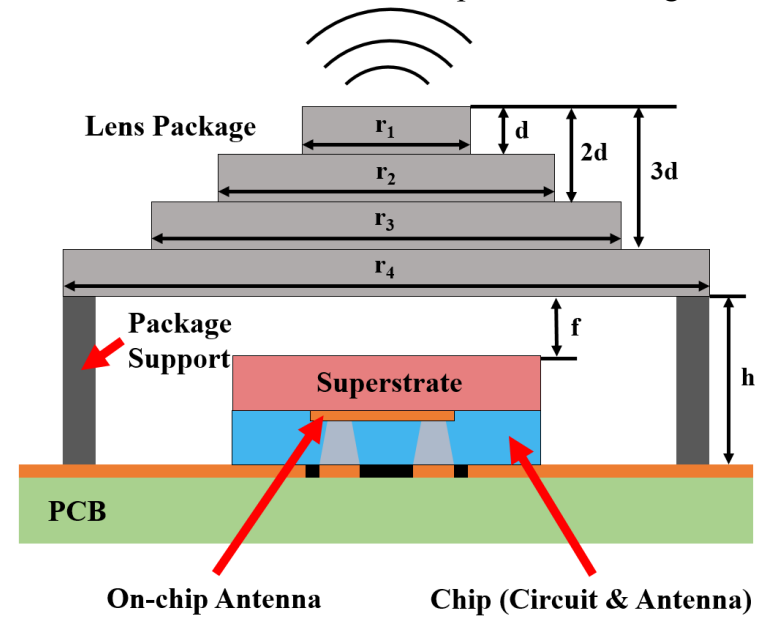

(a)

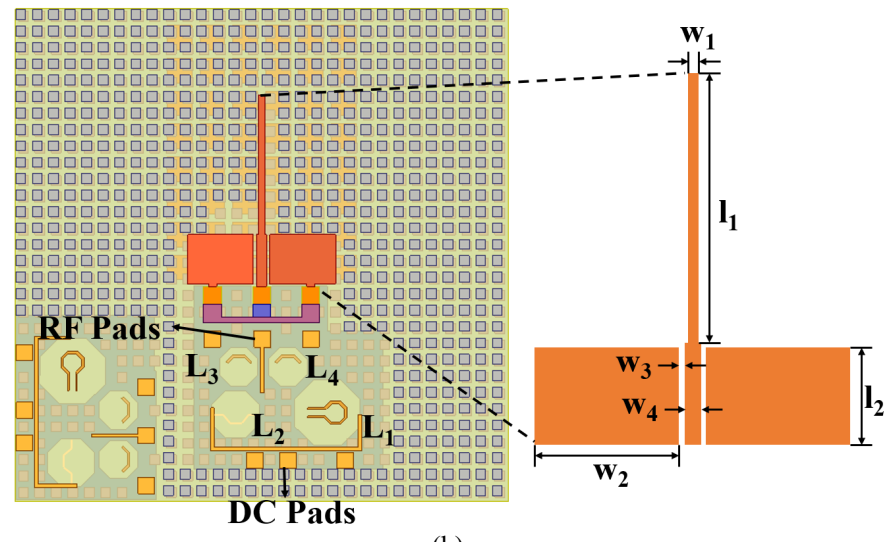

(b)

Fig. 7. HFSS simulation models. (a) The proposed SoP model, $r_{1}=5.46, r_{2}=$ $8.28, r_{3}=10.78, r_{4}=13.16, d=1.44, h=3.26, f=2.7$ (all in mm). (b) The AoC and the configuration of the CPW-fed monopole antenna, chip size: $1.5 \times 1.5$ $\mathrm{mm}^{2} ; w_{1}=20, w_{2}=200, w_{3}=10, w_{4}=30, l_{l}=425$, and $l_{2}=130$ (all in $\left.\mu \mathrm{m}\right)$.

The optimized SoP design demonstrates a simulated return loss of $-19.3 \mathrm{~dB}$ at $71 \mathrm{GHz}$, as shown in Fig. 8 (a), indicating a good impedance match at the frequency of interest. The $10-\mathrm{dB}$ impedance bandwidth is around $9.4 \mathrm{GHz}$, from $64.8 \mathrm{GHz}$ to $74.2 \mathrm{GHz}$. Fig. 8 (b) provides the simulated radiation patterns of the proposed design. It can be seen that the proposed antenna shows a directive boresight radiation with a maximum gain of $8.8 \mathrm{dBi}$, which achieves approximately $22 \mathrm{dBs}$ of gain enhancement in the simulation compared to the standalone AoC. The antenna shows a good symmetric radiation pattern in the $H$-plane, however, there are multiple ripples in the $E$-plane radiation pattern, as shown in Fig. 8 (b), which are mainly caused by the presence of the relatively large ground plane in the driving circuit part. This is also the main reason that the antenna boresight beam is slightly titled. Fig. 8 (c) shows the electric field distribution of the proposed SoP design. The plane wave with a reduced beam width is clearly formed after passing through the Fresnel lens, verifying the beam-focusing ability of the functional package.

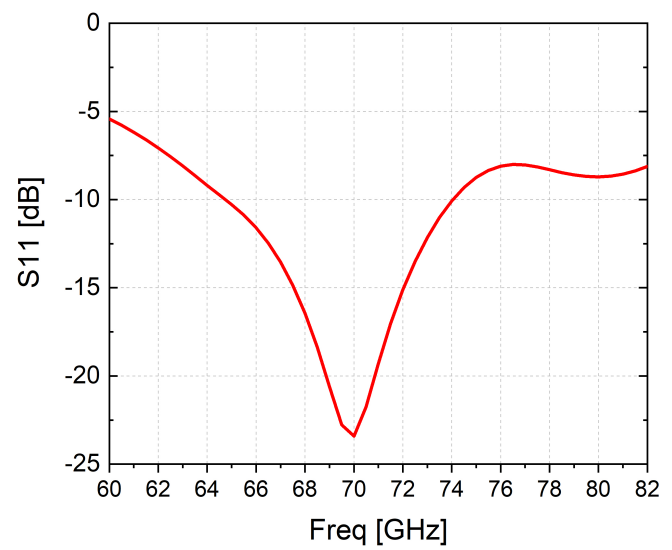

(a) 


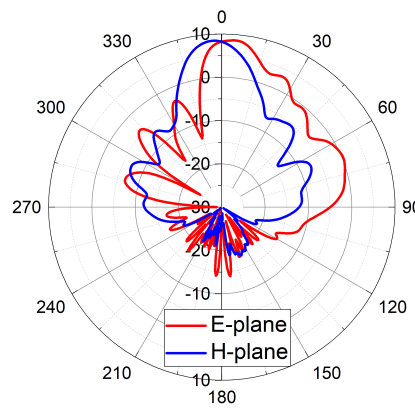

(b)

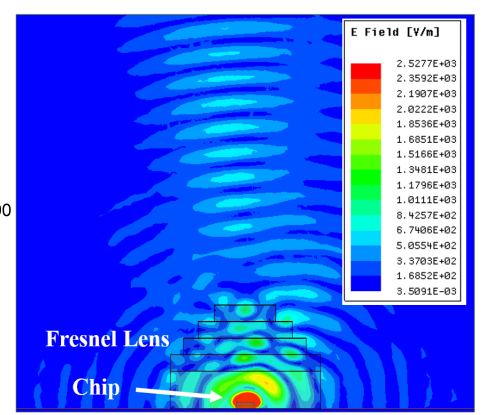

(c)
Fig. 8. HFSS simulation results of the proposed design: (a) return loss versus frequency, (b) 2D radiation pattern ( $E$-plane and $H$-plane), and (c) electric field distribution

\section{ON-ChIP ANTENNA DRIVING CIRCUIT DESIGN}

In real world applications, an on-chip antenna must be fed by the driving circuits, and it must operate well in the presence of circuits, interconnects, bond pads, etc. Therefore, for realistic testing of the on-chip antenna in the presence of circuits, the driving circuits were also designed. Fig. 9 shows the schematic of the on-chip driving circuits, which included a VCO, a frequency doubler, and a matching network that feeds the AoC. The VCO generates a $35.5 \mathrm{GHz}$ RF signal. Since the cutoff frequency of the transistor in the $0.18 \mu \mathrm{m}$ CMOS technology (used for this project due to its lower cost) is $52 \mathrm{GHz}$, the VCO cannot generate a $71 \mathrm{GHz}$ RF signal efficiently. Therefore, a frequency doubler was designed to double the VCO output frequency to $71 \mathrm{GHz}$. The matching network was used to match the impedance between the $\mathrm{AoC}$ and the frequency doubler to ensure maximum power transmission.

Fig. 10 (a) shows the schematic of the VCO. The cross-coupled NMOS transistors $\left(M_{1}\right.$ and $\left.M_{2}\right)$ generate the negative resistance to cater for losses from the LC tank $\left(L_{l}, C_{l}\right.$, and $C_{2}$ ). By tuning the control voltage $V_{\text {tune }}$, the capacitance of the two varactors $\left(C_{1}\right.$ and $\left.C_{2}\right)$ can be adjusted accordingly. As a result, the oscillating frequency of the LC tank can be tuned. The VCO outputs a $35.5 \mathrm{GHz}$ RF signal at the port between $+V_{\text {out }}$ and $-V_{\text {out }}$, which is applied to the port between $V_{\text {in }}+$ and $V_{i n}$ - for the frequency doubler, as shown in Fig.10 (b). The two transistors $\left(M_{5}\right.$ and $\left.M_{6}\right)$ are biased to generate the $2^{\text {nd }}$ order harmonic $(71 \mathrm{GHz})$ at the inductive load $L_{2}$ where the $2^{\text {nd }}$ harmonic and other even harmonics add up constructively while all the odd harmonics cancel with each other. The conventional LC matching network was applied to transform the output impedance of frequency doubler to $50 \Omega$, which is consistent with the AoC input impedance. The simulations of the driving circuit were conducted in Cadence. Fig. 11 depicts the simulated spectrum of the driving circuit output. It can be seen that a $71.2 \mathrm{GHz}$ RF signal with $-12.1 \mathrm{dBm}$ power is obtained at the $2^{\text {nd }}$ order harmonic.

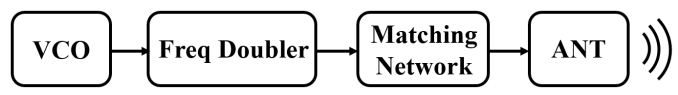

Fig. 9. Schematic of the on-chip system

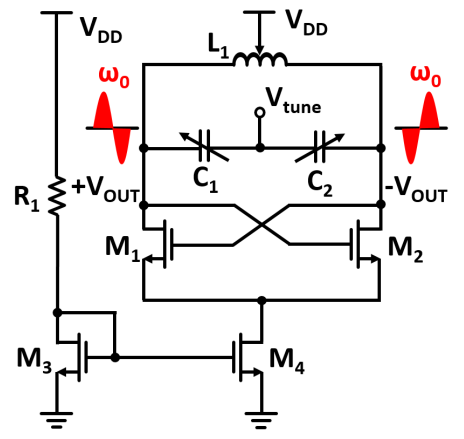

(a)

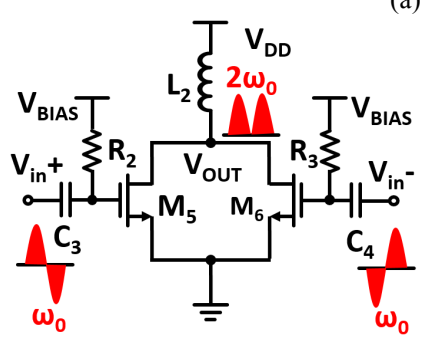

(b)

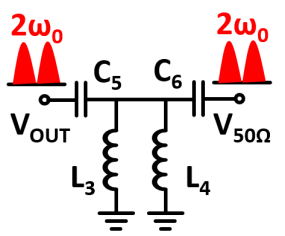

(c)
Fig. 10. Schematic of the (a) VCO, (b) frequency doubler, and (c) matching network

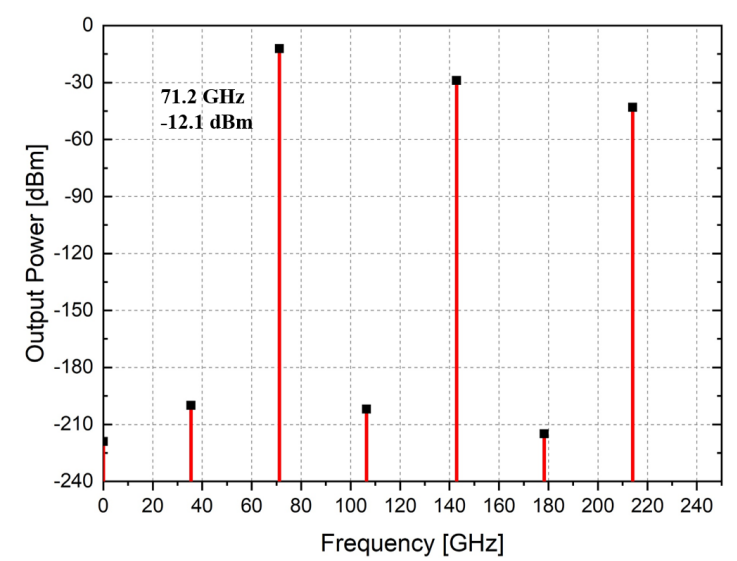

Fig. 11. Frequency spectrum of the driving circuit output

\section{MEASUREMENT AND ANALYSiS}

\section{A. Driving Circuit Measurement}

The driving circuit and AoC were monolithically integrated in a single chip, which has been fabricated in the Taiwan Semiconductor Manufacturing Company (TSMC) $0.18 \mu \mathrm{m}$ CMOS process. The microscopic view of the chip is shown in Fig. 12. The total area of the chip is $1.5 \mathrm{~mm} \times 1.5 \mathrm{~mm}$. The AoC occupies an area of $0.6 \mathrm{~mm} \times 0.8 \mathrm{~mm}$, while the driving circuits occupy an area of $0.56 \mathrm{~mm} \times 0.50 \mathrm{~mm}$. Fig. 13 shows the block diagram of the circuit measurement setup. The driving circuit is powered up, and its output is connected to the spectrum analyzer through the RF signal path, which consists of the RF probe, the RF cables, and the mixer. The uncalibrated power spectrum was obtained from the spectrum analyzer, after which the loss of the RF signal path mentioned above was calibrated by replacing the driving circuit shown in Fig. 13 with a signal generator. 


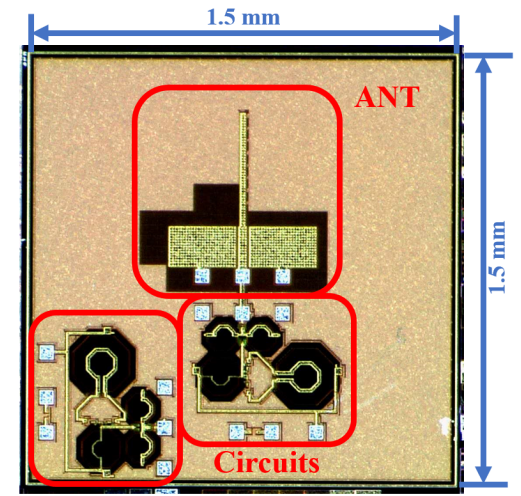

Fig. 12. Microscopic view of the chip

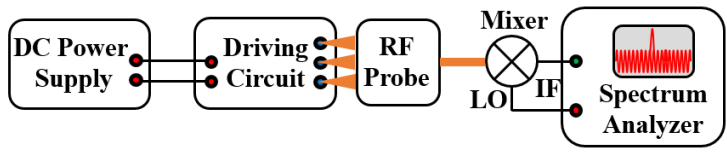

Fig. 13. Block diagram of the driving circuit measurement setup

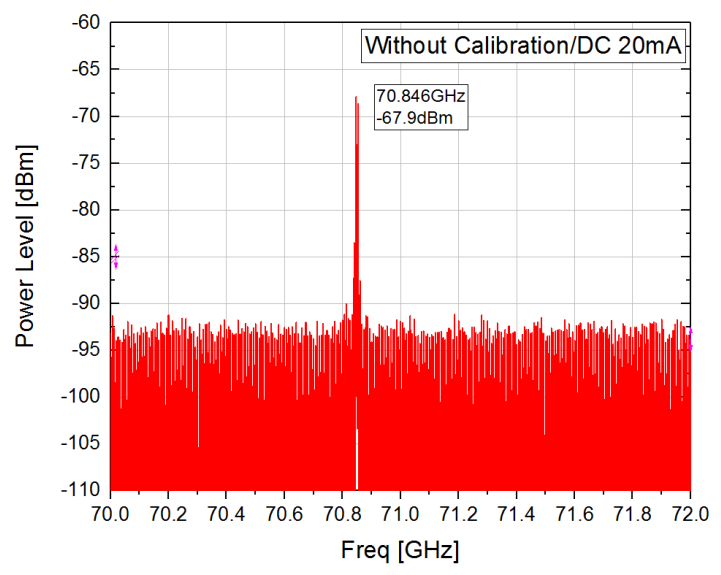

Fig. 14. Driving circuit power spectrum

According to the circuit output power spectrum in Fig. 14, the driving circuit generates a $70.84 \mathrm{GHz} \mathrm{RF}$ signal when a supply voltage of $1.8 \mathrm{~V}$ is applied. The circuit consumes 25.2 $\mathrm{mW}$ of DC power. The power level of the RF signal received by the spectrum analyzer is $-67.9 \mathrm{dBm}$. After calibration, the 54.3 $\mathrm{dB}$ path loss is added back to the measured output power level, so the real circuit output power is $-13.6 \mathrm{dBm}$ at $70.84 \mathrm{GHz}$. This is $1.5 \mathrm{~dB}$ lower than predicted by the simulations. This discrepancy may be due to an error in the manual path loss calibration.

\section{B. On-chip Antenna Passive Measurement and Analysis}

The driving circuit and $\mathrm{AoC}$ are connected through a short transmission line. To characterize the $\mathrm{AoC}$ independently without the circuit's loading effect, the transmission line must be disconnected from the antenna. This was accomplished through the focus ion beam (FIB), as shown in the magnified portion of Fig. 15. The compact mm-wave anechoic chamber shown in Fig. 16 was used to characterize the reflection coefficient and radiation performance of the proposed AoC [27].

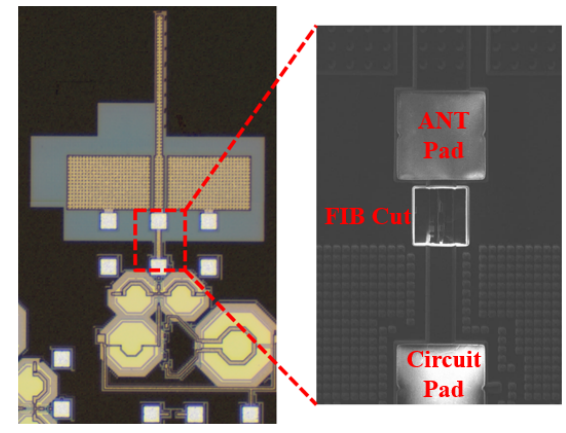

Fig. 15. Transmission line cut by FIB

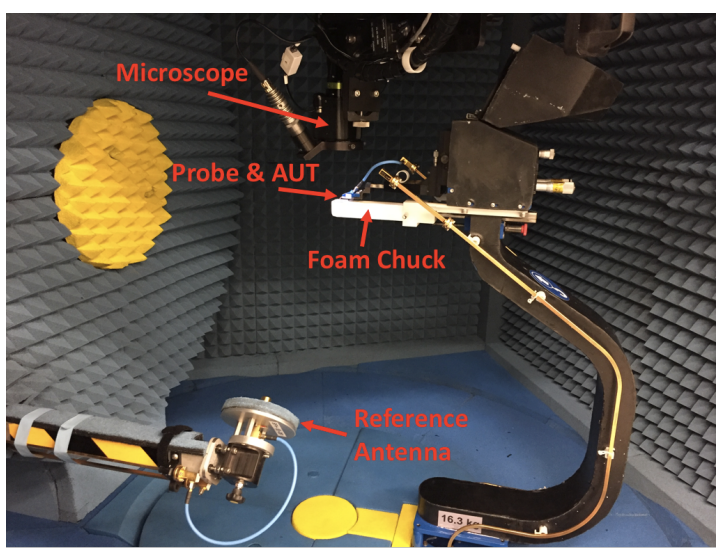

Fig. 16. $\mu$-lab mm-wave anechoic chamber

The antenna under test (AUT) was placed at the center of the chamber on top of a foam chuck to avoid metallization effect from a metal chuck. For this passive testing, the antenna needs to be fed from an RF probe. For that purpose, a set of GSG pads were provided on this chip. The AUT was connected to the power network analyzer (PNA) through the probe and the waveguide. The reference antenna, a horn antenna with a gain of $16 \mathrm{dBi}$ at $71 \mathrm{GHz}$, was connected to the scanning arm and aligned its boresight to the AUT. The microscope and the vision system were used to assist the alignment and landing of the probe with respect to the AUT. Before the radiation pattern measurement, the path loss in this measurement setup was calibrated by directly connecting the two ports with a known loss cable. For the return loss measurement, the probe was calibrated by landing it on the short, open, and load of the impedance standard substrate prior to the $S_{1 I}$ measurement.

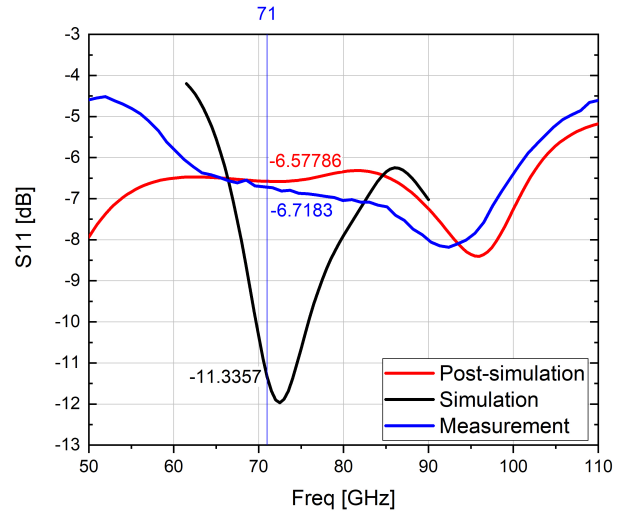

Fig. 17. Simulated and measured return loss of the AoC integrated with the AMC 


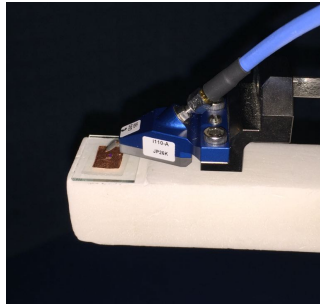

(a)

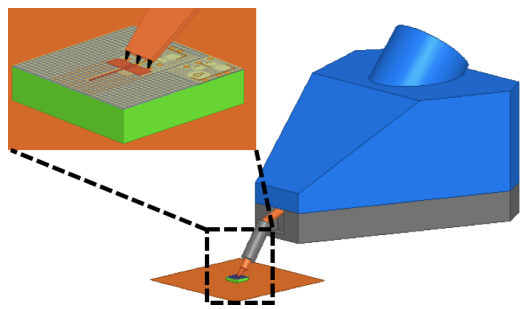

(b)
Fig. 18. (a) Probe landed on the antenna pads. (b) Probe simulation model

Fig. 17 shows the simulated and measured reflection coefficients for the AoC. Some obvious discrepancies exist between the measurement (blue) and simulation (black) results. In the simulations, the antenna is well matched at $71 \mathrm{GHz}$, however, it is not matched well at the frequency of interest in the measurements. After a detailed investigation, we discovered two main reasons for this discrepancy.

- The coupling effects between the AoC and the driving circuits: The AoC and the driving circuits are integrated in a single chip and placed close to each other to reduce transmission loss. The inductors, capacitors, interconnection lines, and dummy metal sheets in the circuit part can lead to unwanted EM coupling when the AoC is excited.

- The probe effect: Although the probe's inner impedance was de-embedded through the calibration, the large metallic body of the probe in close vicinity to the antenna remains a problem. In addition, the probe tips are not completely covered with the absorber, resulting in unwanted coupling between the probe tips and the circuit under the probe [28].

The accurate circuit model, dummy metal fills, and probe model were not included in the initial simulation, therefore, there are major discrepancies between the simulated and measured results. In order to verify the two above-mentioned effects, the accurate circuit model, dummy metal fills, and probe model were included in the post-simulation, as shown in Fig. 18. It can be seen that the post-simulation results (red) show a good agreement with the measurement results (blue) in the range of $60-110 \mathrm{GHz}$. This result verifies the hypothesis that the discrepancy was caused by coupling effects between the antenna and probe as well as some parts of the driving circuits.

Fig. 19 shows the measured and simulated radiation patterns of the AoC with the integrated AMC surface. A measured boresight gain of $-7.3 \mathrm{dBi}$ was obtained. The measured radiation pattern follows similar trends, however, there are some discrepancies between the simulated and measured results. The chamber has a measurement arm (as can be seen in Fig. 16), which blocks the radiation pattern measurements for the range of $40^{\circ} \leq$ theta $\leq 230^{\circ}$ in the E-plane. We anticipate that the main reason for the discrepancy is the presence of the probe near the AoC. The probe tip itself radiates, so the measured radiation patterns are actually the supposition of probe self-radiation and antenna radiation. The probe body is a close-by conductor, and it can reflect the EM waves radiated by the antenna and cause interference at certain angles [29]. Though, we have an approximate probe model in the simulations, but at this frequency, a more accurate model is necessary to capture what occurs in the measurements. In the future, customized mm-wave absorbers could be used to cover the conductor body to avoid EM reflection and decrease the influence of probe tip self-radiation.

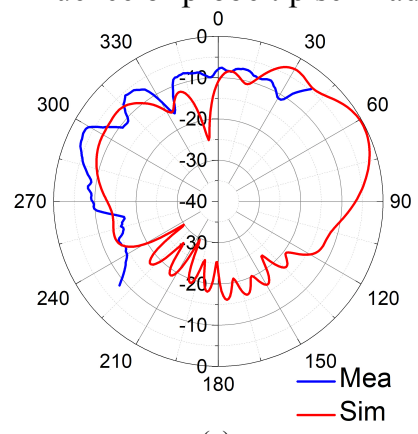

(a)

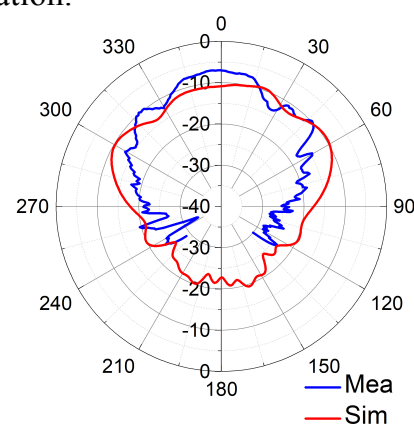

(b)
Fig. 19. Measured and simulated radiation patterns. (a) $E$-plane and (b) $H$-plane

\section{Active Measurements for SoP Implementation}

The functional package was fabricated through additive manufacturing technologies, including inkjet and 3D printing. The DC connections between the on-chip circuit pads and the printed circuit board (PCB) were fabricated through inkjet printing technology instead of traditional bond wiring technology. This absence of bond wires enables a smooth attachment of the superstrate layer to the chip. A dielectric isolation layer was first inkjet printed on top of the on-chip circuit area to avoid short circuiting the printed DC connections with the circuits. The DC connections (ground and power supply) were printed from the on-chip pads to the edge of the chip, as shown in Fig. 20. Subsequently, a silver paste was applied to connect the chip edge to the PCB, as shown in Fig. 21(a). A $300 \mu \mathrm{m}$ layer of PREFERM FLX 1100 was used as the superstrate layer. The Fresnel lens package and its support shown in Fig. 21(b) were fabricated through 3D printing technology with the PREFERM 3D ABS filament $\left(\varepsilon_{r}=10, \tan \delta\right.$ $=0.02$ at $71 \mathrm{GHz}$ ).
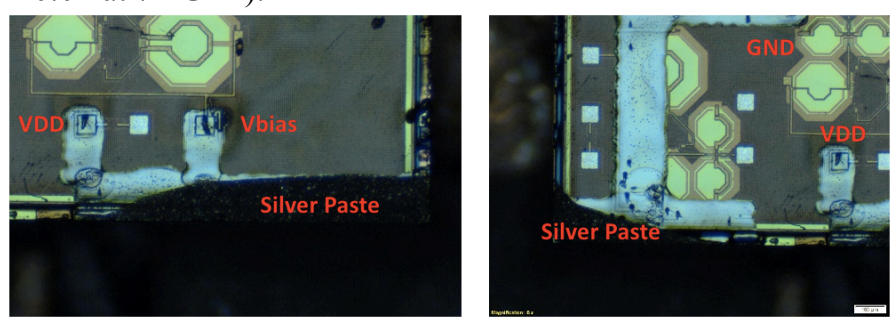

Fig. 20. Inkjet printed on-chip DC connection lines

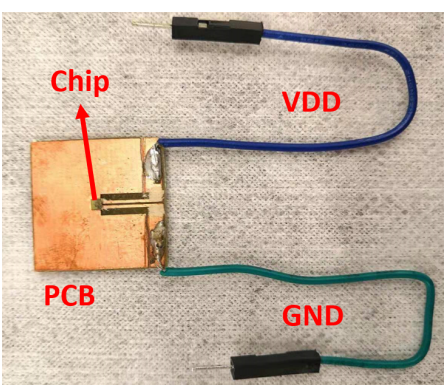

(a)

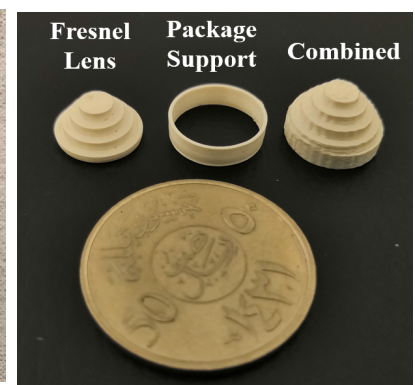

(b)
Fig. 21. (a) DC connection of a 3D-printed chip package. (b) Additively manufactured Fresnel lens package 
Once the chip is packaged (with an additively manufactured Fresnel lens), the GSG pads are not accessible for probe-based measurements, so active measurements were performed to assess the gain enhancement performance as predicted by the simulations. Fig. 22 illustrates the dedicated setup used for active measurements as these could not be performed conventionally in the chamber.

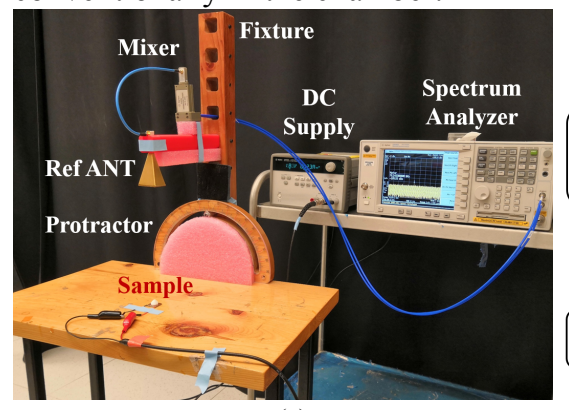

(a)

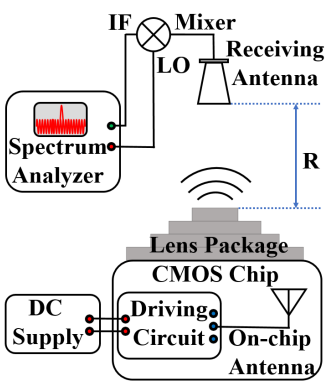

(b)
Fig. 22. AoC active measurement setup: (a) photograph and (b) block diagram

For active measurements, the driving circuit is first powered up by the DC power supply. The driving circuit generates the $\mathrm{RF}$ signal with a power level of $-13.6 \mathrm{dBm}$ at $70.84 \mathrm{GHz}$, which is fed to the $\mathrm{AoC}$ as the transmitted power. The receiving antenna is a V-band reference horn antenna with a gain of 24 $\mathrm{dBi}$ at $50-75 \mathrm{GHz}$. The receiving antenna and the AUT are aligned in a boresight-to-boresight configuration with a distance of $R=30 \mathrm{~cm}$, which is larger than $r=2 D^{2} / \lambda_{0}$, satisfying the far-field condition. The antenna's largest aperture dimension and free-space wavelength at $71 \mathrm{GHz}$ are $D$ and $\lambda_{0}$, respectively. The RF signal received by the reference horn antenna is then transmitted to the spectrum analyzer through $R F$ cables and the mixer with a total path loss $L$ of $51.2 \mathrm{~dB}$ at the operating frequency. The received power is measured by the Agilent E4448A spectrum analyzer. The absolute gain of the AUT is obtained by applying the Friis transmission equation, as shown below:

$$
G_{t}=P_{r}-P_{t}-G_{r}-20 \log _{10}\left(\frac{\lambda_{0}}{4 \pi R}\right)-L
$$

Where $G_{t}$ and $G_{r}$ are the gains of the transmitting antenna (AUT) and the reference horn antenna, $P_{r}$ and $P_{t}$, are the received and transmitted power, respectively. $L$ is the total path loss and the $R$ is the boresight distance between the AUT and the reference antenna.

Fig. 23 shows the measured radiation patterns of the $E$ - and $H$-planes compared to the simulated radiation patterns for all four cases: the AoC integrated with the AMC; the AoC integrated with the $\mathrm{AMC}$ and superstrate; the $\mathrm{AoC}$ integrated with the AMC and Fresnel lens; and the AoC integrated with the AMC, superstrate, and Fresnel lens. These four cases were simulated and measured for investigating the gain enhancement performance of the AMC, superstrate, and Fresnel lens. Due to the constraints of the active measurement setup, the measurements of the radiation patterns were only performed for the range of $-50^{\circ} \leq$ theta $\leq 50^{\circ}$. As shown in Fig. 23, there is acceptable agreement between the simulated and measured radiation patterns. It can be seen that the final SoP (the AoC integrated with the AMC, superstrate, and Fresnel lens) displays the narrowest beam width compared to other cases.

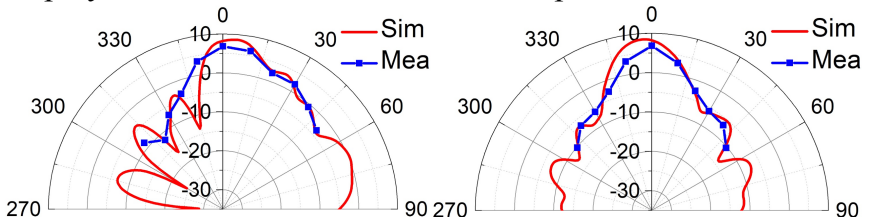

(a)

(b)

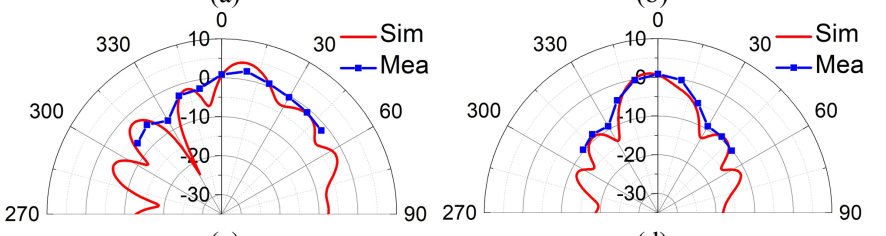

(c)

(d)

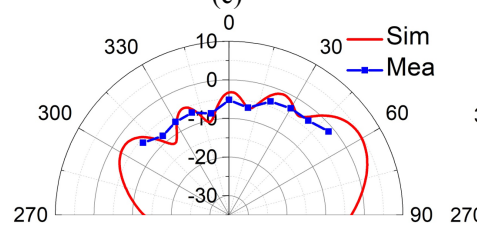

(e)

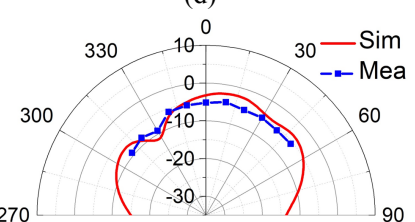

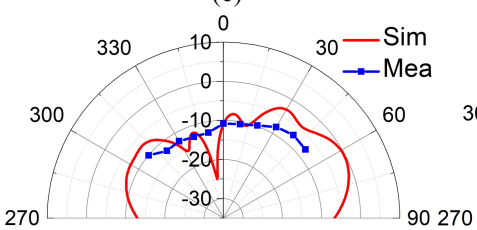

(g) (f)

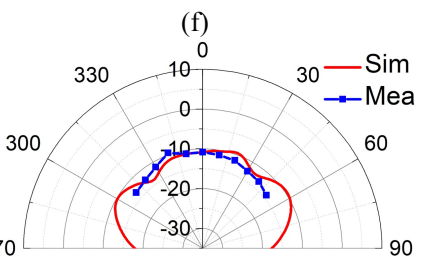

(h)
Fig. 23. Simulated and measured radiation patterns in E-plane (left) and H-plane (right). (a)(b) AoC integrated with the AMC, superstrate, and Fresnel lens; (c)(d) AoC integrated with the AMC and Fresnel lens; (e)(f) AoC integrated with the AMC and superstrate; $(\mathrm{g})(\mathrm{h})$ AoC integrated with the AMC

TABLE I. THE COMPARISON OF RADIATION PERFORMANCE

\begin{tabular}{|c|c|c|c|}
\hline \multirow{2}{*}{ Cases } & \multicolumn{3}{|c|}{ AoC Boresight Radiation Performance } \\
\cline { 2 - 4 } & Description & Simulation & Measurement \\
\hline $\mathbf{1}$ & Standalone AoC & $-13.8 \mathrm{dBi}$ & $\mathrm{NA}$ \\
\hline $\mathbf{2}$ & AoC + AMC & $-10.8 \mathrm{dBi}$ & $-9.7 \mathrm{dBi}$ \\
\hline $\mathbf{3}$ & AoC + AMC + superstrate & $-3.2 \mathrm{dBi}$ & $-5.2 \mathrm{dBi}$ \\
\hline $\mathbf{4}$ & AoC + AMC + Fresnel lens & $0.6 \mathrm{dBi}$ & $0.8 \mathrm{dBi}$ \\
\hline $\mathbf{5}$ & $\begin{array}{c}\text { AoC + AMC + superstrate } \\
\text { + Fresnel lens }\end{array}$ & $8.3 \mathrm{dBi}$ & $6.8 \mathrm{dBi}$ \\
\hline
\end{tabular}

Table I summarizes the boresight radiation performance for all the cases in the measurements and simulations. The combination of AMC, superstrate, and Fresnel lens enhanced the gain by $\sim 20 \mathrm{dBs}$ compared to the AoC alone. This is a very large enhancement in gain considering the fact that this is coming from the package of the chip, which would not add anything to the gain in normal circumstances. As can be seen from Table I, the Fresnel lens provides around $12 \mathrm{dBs}$ of gain enhancement, if we compare the gains in Case (3) and Case (5). In addition, according to the comparison between Case (2) and Case (3), around $7 \mathrm{dBs}$ of gain enhancement is obtained by using the superstrate layer. Comparing the simulation results of Case (1) and Case (2) reveals that the AMC adds up to $3 \mathrm{dBs}$ of gain compared to the standalone AoC. The standalone AoC gain measurement could not be performed because the AMC is built into the chip during the chip fabrication process. 


\section{CONCLUSION}

The AoC has been considered an excellent solution for mm-wave applications that require high integration levels and low costs. However, the AoC suffers from low gain because of the lossy Si substrate. In addition, it is well known that all chips need to be packaged. In mm-wave ranges, the presence of the chip package can further deteriorate the AoC performance. This paper proposes one method to enhance the gain by shaping the chip package into a specially designed functional package structure, which combines a superstrate layer and a Fresnel lens. This functional package can improve the antenna's radiation performance as well as provide chip protection. Furthermore, an on-chip AMC surface is also used to enhance the AoC gain. In this work, a $71 \mathrm{GHz} \mathrm{CPW}$ fed on-chip monopole antenna was implemented in a $0.18 \mu \mathrm{m}$ standard CMOS process. The proposed AoC integrated with an AMC and functional package achieved a measured gain of $6.8 \mathrm{dBi}$ at $71 \mathrm{GHz}$ with an impedance bandwidth of $9.2 \mathrm{GHz}$. The gain enhancement of the overall combination was experimentally demonstrated to be $20.6 \mathrm{~dB}$, indicating a substantial gain enhancement over a standalone AoC.

\section{ACKNOWLEDGMENT}

The authors would like to thank Dr. Yen-Hung Kuo for his assistance with the IC design and chip layout.

\section{REFERENCES}

[1] O. K. K et al., "On-chip antennas in silicon ICs and their application," IEEE Transactions on Electron Devices, vol. 52, no. 7, pp. 1312-1323, 2005.

[2] H. M. Cheema and A. Shamim, "The last barrier: on-chip antennas," IEEE Microwave Magazine, vol. 14, no. 1, pp. 79-91, 2013.

[3] M. Nafe, A. Syed, and A. Shamim, "Gain-Enhanced On-Chip Folded Dipole Antenna Utilizing Artificial Magnetic Conductor at $94 \mathrm{GHz}$," IEEE Antennas and Wireless Propagation Letters, vol. 16, pp. 2844-2847, 2017.

[4] S. Pan, L. Gilreath, P. Heydari, and F. Capolino, "Investigation of a Wideband BiCMOS Fully On-Chip \$W\$-Band Bowtie Slot Antenna," IEEE Antennas and Wireless Propagation Letters, vol. 12, pp. 706-709, 2013.

[5] F. A. Ghaffar, M. Arsalan, H. M. Cheema, K. N. Salama, and A. Shamim, "60 GHz system-on-chip (SoC) with built-in memory and an on-chip antenna," in The 8th European Conference on Antennas and Propagation (EuCAP 2014), 2014, pp. 531-532.

[6] P. Burasa, T. Djerafi, N. G. Constantin, and K. Wu, "On-Chip Dual-Band Rectangular Slot Antenna for Single-Chip Millimeter-Wave Identification Tag in Standard CMOS Technology," IEEE Transactions on Antennas and Propagation, vol. 65, no. 8, pp. 3858-3868, 2017.

[7] Y. Song et al., "A Hybrid Integrated High-Gain Antenna With an On-Chip Radiator Backed by Off-Chip Ground for System-on-Chip Applications," IEEE Transactions on Components, Packaging and Manufacturing Technology, vol. 7, no. 1, pp. 114-122, 2017.

[8] M. S. Khan, F. A. Tahir, A. Meredov, A. Shamim, and H. M. Cheema, "A W-Band EBG-Backed Double-Rhomboid Bowtie-Slot On-Chip Antenna," IEEE Antennas and Wireless Propagation Letters, vol. 18, no. 5, pp. 1046-1050, 2019.

[9] K. Ming-Dou and H. Sheng-Fu, "Methodology on Extracting Compact Layout Rules for Latchup Prevention," in Transient-Induced Latchup in CMOS Integrated Circuits: IEEE, 2009, p. 1.

[10] T. Deng, Z. Chen, and Y. P. Zhang, "Coupling Mechanisms and Effects Between On-Chip Antenna and Inductor or Coplanar Waveguide," IEEE Transactions on Electron Devices, vol. 60, no. 1, pp. 20-27, 2013.

[11] I. Papapolymerou, R. F. Drayton, and L. P. B. Katehi, "Micromachined patch antennas," IEEE Transactions on Antennas and Propagation, vol. 46, no. 2, pp. 275-283, 1998.
[12] K. T. Chan et al., "Integrated antennas on $\mathrm{Si}$ with over $100 \mathrm{GHz}$ performance, fabricated using an optimized proton implantation process," IEEE Microwave and Wireless Components Letters, vol. 13, no. 11, pp. 487-489, 2003.

[13] A. Babakhani, X. Guan, A. Komijani, A. Natarajan, and A. Hajimiri, "A 77-GHz Phased-Array Transceiver With On-Chip Antennas in Silicon: Receiver and Antennas," IEEE Journal of Solid-State Circuits, vol. 41, no. 12, pp. 2795-2806, 2006.

[14] S. Pan, D. Wang, C. Guclu, and F. Capolino, "High impedance layer for CMOS on-chip antenna at millimeter waves," in 2011 IEEE International Symposium on Antennas and Propagation (APSURSI), 2011, pp. 903-906.

[15] X. Bao, Y. Guo, and Y. Xiong, "60-GHz AMC-Based Circularly Polarized On-Chip Antenna Using Standard 0.18-\$mu\$m CMOS Technology," IEEE Transactions on Antennas and Propagation, vol. 60, no. 5, pp. 2234-2241, 2012.

[16] H. Chu, Y. Guo, T. Lim, Y. M. Khoo, and X. Shi, "135-GHz Micromachined On-Chip Antenna and Antenna Array," IEEE Transactions on Antennas and Propagation, vol. 60, no. 10, pp. 4582-4588, 2012.

[17] J. M. Edwards and G. M. Rebeiz, "High-Efficiency Elliptical Slot Antennas With Quartz Superstrates for Silicon RFICs," IEEE Transactions on Antennas and Propagation, vol. 60, no. 11, pp. 5010-5020, 2012.

[18] L. Marnat, A. A. A. Carreno, D. Conchouso, M. G. Martı'nez, I. G. Foulds, and A. Shamim, "New Movable Plate for Efficient Millimeter Wave Vertical on-Chip Antenna," IEEE Transactions on Antennas and Propagation, vol. 61, no. 4, pp. 1608-1615, 2013.

[19] R. Wu et al., "A 60-GHz efficiency-enhanced on-chip dipole antenna using helium-3 ion implantation process," in 2014 44th European Microwave Conference, 2014, pp. 108-111.

[20] W. T. Khan et al., "A D-Band Micromachined End-Fire Antenna in 130-nm SiGe BiCMOS Technology," IEEE Transactions on Antennas and Propagation, vol. 63, no. 6, pp. 2449-2459, 2015.

[21] H. Zhang and A. Shamim, "Gain and Efficiency Enhancement of a 77 GHz On-Chip Antenna through AMC and Superstrate Package," in 2018 IEEE International Symposium on Antennas and Propagation \& USNC/URSI National Radio Science Meeting, 2018, pp. 363-364.

[22] B. S. Cook and A. Shamim, "Utilizing Wideband AMC Structures for High-Gain Inkjet-Printed Antennas on Lossy Paper Substrate," IEEE Antennas and Wireless Propagation Letters, vol. 12, pp. 76-79, 2013.

[23] D. Jackson and N. Alexopoulos, "Gain enhancement methods for printed circuit antennas," IEEE Transactions on Antennas and Propagation, vol. 33, no. 9, pp. 976-987, 1985.

[24] F. A. Ghaffar, M. U. Khalid, K. N. Salama, and A. Shamim, "24-GHz LTCC Fractal Antenna Array SoP With Integrated Fresnel Lens," IEEE Antennas and Wireless Propagation Letters, vol. 10, pp. 705-708, 2011.

[25] D. N. Black and J. C. Wiltse, "Millimeter-Wave Characteristics of Phase-Correcting Fresnel Zone Plates," IEEE Transactions on Microwave Theory and Techniques, vol. 35, no. 12, pp. 1122-1129, 1987.

[26] A. Petosa and A. Ittipiboon, "Design and performance of a perforated dielectric Fresnel lens," IEE Proceedings - Microwaves, Antennas and Propagation, vol. 141, no. 5, p. 309, 1994.

[27] H. Zhang and A. Shamim, "Tackling the Issues of Millimeter-wave On-chip Antenna Measurements," in 2019 13th European Conference on Antennas and Propagation (EuCAP), 2019, pp. 1-5.

[28] M. Seyyedesfahlan and I. Tekin, "ACP Probe Measurement of On-Chip Strip Dipole Antennas at W Band," IEEE Transactions on Antennas and Propagation, vol. 64, no. 4, pp. 1270-1278, 2016.

[29] A. C. F. Reniers, A. R. v. Dommele, A. B. Smolders, and M. H. A. J. Herben, "The Influence of the Probe Connection on mm-Wave Antenna Measurements," IEEE Transactions on Antennas and Propagation, vol. 63, no. 9 , pp. 3819-3825, 2015.

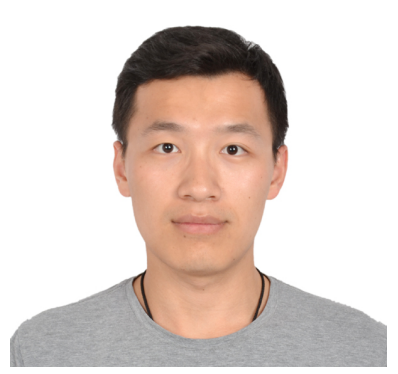

Haoran Zhang was born in Taian, China. He received the Bachelor degree of electrical engineering from University of Electronic Science and Technology of China (UESTC), Chengdu, China in 2017 and Master degree of electrical engineering from King Abdullah University Science and 
Technology (KAUST), Saudi Arabia in 2019.

From 2019 till now, he is a Ph.D. candidate working in IMPACT Lab, Electrical Engineering program, KAUST, Saudi Arabia. His research interests include mm-wave on-chip antenna design, antenna array design for $5 \mathrm{G}$ applications, and electrically small antenna design.

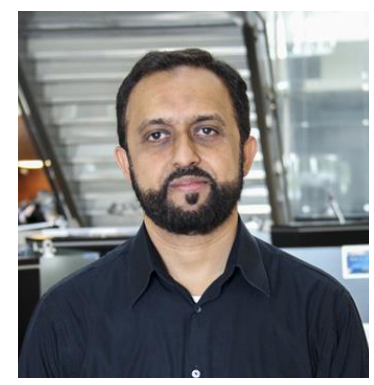

Atif Shamim (SM'13) received his M.A.Sc. and Ph.D degrees in electrical engineering at Carleton University, Canada in 2004 and 2009 respectively. He was an NSERC Alexander Graham Bell Graduate scholar at Carleton University from 2007 till 2009 and an

NSERC postdoctoral Fellow in 2009-2010 at Royal Military College Canada and KAUST. In August 2010, he joined the Electrical Engineering Program at KAUST, where he is currently an Associate Professor and principal investigator of IMPACT Lab. He was an invited researcher at the VTT Micro-modules Research Center (Oulu, Finland) in 2006. Dr. Shamim was the recipient of the best paper prize at the European Microwave Association Conference in 2008. He was given the Ottawa Centre of Research Innovation (OCRI) Researcher of the Year 2008 Award in Canada. His work on Wireless Dosimeter won the ITAC SMC Award at Canadian Microelectronics Corporation TEXPO in 2007. Prof. Shamim's students won the Best Paper Prize (3rd position) in IEEE IMS 2016 and (1st position) in IEEE MECAP 2016. Prof. Shamim also won numerous business-related awards, including the 1st prize in Canada's national business plan competition and was selected for OCRI Entrepreneur of the year award in 2010. He is an author/co-author of over 180 international publications and an inventor on 20 patents. His research interests are in innovative antenna designs and their integration strategies with circuits and sensors for flexible and wearable wireless sensing systems through a combination of CMOS and additive manufacturing technologies. Dr. Shamim is a Senior Member of IEEE and serves on the editorial board of IEEE Transactions on Antennas and Propagation. 